\title{
observaciones acerca de la determinación de alúmina en cementos
}

Jose CALLEJ A CARHETE, Doctor en Ciencias Químicas, y J. MANdeI, FERnandez Paris, Perito Químico.

La alúmina (óxido alumínico) es uno de los componentes principales del clínker y del cemento portland, cuya determinación tiene interés para el conocimiento de las propiedades y comportamiento del cemento, sobre todo en el aśpecto del fraguado, del calor de hidratación y de la durabilidad. También interesa como dato de partida para el cálculo de la composición potencial, y en tal sentido las Normas A.S.T.M. prescriben que el resultado analítico de la alúmina se exprese con dos cifras decimales, es decir, con aproximación de 0,01 (lo mismo que el resultado analítico del óxido fértico $y$, eventualmente, los de $\mathrm{TiO}_{2}$ y $\mathrm{P}_{2} \mathrm{O}_{5}$ ) (1).

El cálculo de la composición potencial del clínker y del cemento portland por el método de Bogue (2) es sólo aproximado, por cuanto que se basa en una serie de hipótesis que, en la realidad, no se cumplen sino de una manera también aproximada.

La determinación de la alúmina por el método tradicional de las Normas A.S.T.M., es decir, hallando gravimétricamente el conjunto de óxidos $\mathrm{R}_{x} \mathrm{O}_{y}$ de los elementos que forman parte del llamado «grupo del hidróxido amónico» (1), esto es, los sesquióxidos, $\mathrm{R}_{2} \mathrm{O}_{3}\left(\mathrm{Al}_{2} \mathrm{O}_{3}+\mathrm{Fe}_{2} \mathrm{O}_{3}\right)$, el bióxido de titanio, $\mathrm{TiO}_{3}, \mathrm{y}$ el pentóxido de fósforo, $\mathrm{P}_{2} \mathrm{O}_{5}, \mathrm{y}$ restando de dicho conjunto los valores correspondientes a $\mathrm{Fe}_{2} \mathrm{O}_{3}, \mathrm{TiO}_{2}$ y $\mathrm{P}_{2} \mathrm{O}_{5}$, hallados aparte, implica la necesidad de conocer estos valores, de los cuales el de $\mathrm{Fe}_{2} \mathrm{O}_{3}$ se determina en los análisis de rutina de los cementos. pero no así los de $\mathrm{TiO}_{2} \mathrm{y}$, sobre todo, $\mathrm{P}_{2} \mathrm{O}_{5}{ }^{\circ}$.

Esta necesidad se comprende si se tiene en cuenta, por una parte, que en el cálculo de la composición potencial interviene el concepto analítico $\mathrm{Al}_{2} \mathrm{O}_{3}$, el cual, como queda indicado, ha de tomarse con aproximación de $0,0 \mathrm{I}, \mathrm{y}$, por otra, que los contenidos normales de $\mathrm{TiO}_{2} y$ $\mathrm{P}_{2} \mathrm{O}_{5}$ de los cernentos portland usuales suelen oscilar entre 0,20 y 0,30 , y alrededor de $0,05 \%$, respectivamente (4). Además, sólo así tiene sentido exigir para el concepto $\mathrm{Al}_{2} \mathrm{O}_{n}$ (y lo mismo para $\mathrm{Fe}_{2} \mathrm{O}_{3}, \mathrm{TiO}_{2}$ y $\mathrm{P}_{2} \mathrm{O}_{5}$ ) una aproximación de 0,01 .

Otros métodos más modernos, no tradicionales, determinan también la alúmina por diferencia, y generalmente lo hacen, no como alúmina pura, $\mathrm{Al}_{2} \mathrm{O}_{3}$ (óxido alumínico), sino como «alúmina brutas, es decir, como suma de $\mathrm{Al}_{2} \mathrm{O}_{3}$ y $\mathrm{TiO}_{2}$, puesto que en determinadas condiciones queda descartado el $\mathrm{P}_{2} \mathrm{O}_{5}$. Los métodos a que se hace referencia son, concretamente, los complexométricos (5). En éstos, y por las mismas razones ya expuestas, es también imprescindible la determinación de $\mathrm{TiO}_{2}$.

* En las condiciones operatorias de las Normas A.S.T.M.: precipitaclón a ebullictón con hidróxido amónico a pH 7, en presencla de una concentración elevada de cloruro amónico, y fltractón rápida en caliente, no se

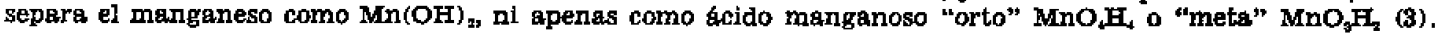


Pero frente a los procedimientos gravimétricos tradicionales, los complexométricos, aparie de su mayor sencillez y rapidez, presentan otra gran ventaja: la de ser susceptibles de dar el valor de la alúmina como tal (óxido alumínico, $\mathrm{Al}_{2} \mathrm{O}_{3}$ ), sin necesidad de determinar aparte $n i \mathrm{TiO}_{2} n i \mathrm{P}_{2} \mathrm{O}_{\xi}$.

En efecto, en un método complexométrico ya propuesto (5), se determina $\mathrm{Al}_{2} \mathrm{O}_{3}$ junto con $\mathrm{TiO}_{n}$ y $\mathrm{Mn}_{2} \mathrm{O}_{3}$ ", y separado de $\mathrm{P}_{2} \mathrm{O}_{5}$. Lo primero, porque en la disolución de la muestra-problema se encuentran $\mathrm{Al}^{\mathrm{s}+}$. $\mathrm{Ti}^{3+}$ y $\mathrm{Mn}^{2+}$, que forman complejos con el reactivo EDTA (sal disódica dihidratada del ácido etiłendiaminotetracético:Complexona III) en las condiciones de trabajo ${ }^{\circ}$; lo segundo, porque en dichas condiciones de trabajo no forma complejos el fósforo ${ }^{* 0}$.

La razón estriba en que en el método indicado se opera directamente sobre la disolución clorhídrica de la muestra problema, sin separación de ningún elemento.

Pero cuando después de una previa separación de sílice por insolubilización se precipitan los elementos del «grupo del hidróxido amónico», se separan el aluminio, el hierro, el titanio y el fósforo, y al redisolver el conjunto de los precipitados, queda sin pasar a la disolución el titanio cuando el tratamiento se hace con ácido clorhídrico, disolviéndose tan sólo cuando el tratamiento de los precipitados se hace con ácido sulfúrico ${ }^{* \circ}$. Por lo tanto, en el líquido filtrado sólo se forman los complejos de los elementos presentes que puedan dar lugar a ellos, es decir, los del hierro y del aluminio.

Para poder poner de relieve estos hechos de forma experimental. se efectuaron tres series de experiencias cuyos resultados se destacan a continuación:

\section{Primera serie}

a) Se intentó preparar una disolución de cloruro titánico $\mathrm{Cl}_{4} \mathrm{Ti}$ por precipitación de otra de sulfato titánico $\left(\mathrm{SO}_{4}\right)_{2} \mathrm{Ti}$, con cloruto bárico. Se filtró el sulfato bárico. Tratado el filtrado con exceso de EDTA en las condiciones de formación del complejo de aluminio, EDTA-Al, no se consiguió formar el complejo de titanio, EDTA-Ti, como demostró la valoración por retorno del exceso (en este caso la totalidad) de EDTA añadido, con disolución patrón de zinc e indicador de «ditizona» (5).

b) Se preparó una disolución de cloruro de aluminio a partir de aluminio purísimo disuelto en ácido clorhídrico reactivo, y con ella, operando como en a), se consiguió la formación total del complejo de aluminio.

c) Con mezclas de partes alícuotas de las disoluciones preparadas según $a$ ) y $b$, se consiguió la formación total del complejo de aluminio, siendo nula la del de titanio.

* De todos modos, la formación de complejo por parte del manganeso no es cuantitativa (6). Esto no tiene importancia ni supone grandes errores, porque el contenido de manganeso del clínker, $y$ aún del cemento portland ordinario, no suele exceder de $0,05 \%$ (4).

* Buena prueba de ello es que cuando se determinan fosfatos por complexometria se recurre al procedimiento indirecto de preclpitar fosfato amónico magnésico y determinar complexométricamente magnesio (7).

* * En reqlidad, en la preclpitación con hidróxido amónico en caliente se forma acido metatitánico, TiO $\mathrm{H}_{2}$. que, a diferencla del ortotitánico, Ti(OH), que precipita en frio, es diffellmente soluble en acidos diluidos $y$, como el $\mathrm{T}_{1} \mathrm{O}_{2}$, mucho más fácilmente soluble en ácido sulfúrico que en foldo clorhidrico (3). 
d) Al tratar la disolución obtenida según a) con peróxido de hidrógeno e inteniar después la formación del complejo EDTA-Ti, no desapareció el color amarillo del compuesto peroxídico y tampoco se observó viraje del indicador «dirizona», probablemente por alteración de ésta provocada por el peróxido de hidrćgeno. No es, en cambio, admisible pensar en la alteración del reactivo EDTA (la otra alternativa posible), por cuanto que el agua oxigenada se utíliza para enmascarar el titanio cuando en presencia de éste se desea valorar complexométricamente zinc, empleando «negro de eriocromo T» como indicador (8). Igualmente existe un método de valoración complexométrica de magnesio cuando se halla junto con calcio, previa separación de éste por precipitación con oxalato amónico y destrucición del exceso de oxalato mediante peróxido de hidrógeno. En este caso se utiliza también como indicador «negro de eriocromo T) (9). En consecuencia, por lo que se refiere al indicador «ditizona», no pudo determinarse si el titanio formó complejo o no, o si lo hizo parcialmente, aun cuando el hecho de que el peróxido de hidrógeno lo enmascara frente al EDTA (8) permite asegurar que no formó complejo.

e) Se tomaron partes alícuotas de la disolución inicial de sulfato titánico y de la de cloruro de aluminio obtenida según $b$ ). Se precipitaron los hidróxidos $\mathrm{R}_{x} \mathrm{O}_{*}\left[\mathrm{Al}(\mathrm{OH})_{3}+\mathrm{Ti}(\mathrm{OH})_{4}\right.$ o más bien $\left.\mathrm{Al}(\mathrm{OH})_{3}+\mathrm{TiO}_{3} \mathrm{H}_{2}\right]$ por el procedimiento ordinario, con hidróxido amónico concentrado y en caliente; se separaron los precipitados por filtración; se lavaron y se redisolvieron en ácido sulfúrico concentrado, volviendo a filtrar. Con la disolución obtenida se intentéto la formación de łos complejos EDTA-Aì y EDTA-Ti en las condiciones apropiadas (5) y se consiguió la formación cuantativa de ambos.

f) Se procedió como en $e$ ), sin más diferencia que la de tratar los precipitados con ácido clorhídrico en lugar de sulfúrico. En este caso se formó exclusivamente el complejo de altuminio, lo que indica que el precipitado de ácido metatitánico no se disuelve con el ácido clorhídrico y permanece en el filtro. En cambio, en el caso e) se disuelve en el ácido sulfúrico.

g) Puesto que los resultados e) y $f$ ) parecen indicar que el titanio forma complejo cuando se encuentra en forma de sulfato, incluso que su disolución se consigue mucho mejor y más completamente en un medio sulfúrico, se operó directamente con la disolución patrón de sulfato titánico y se logró también la formación ctuantitativa del complejo EDTA-Tí.

\section{Segunda serie}

Se efectuaron determinaciones de alúmina en cementos, tanto por el método gravimétrico clásico y tradicional de las Normas A.S.T.M. (1), como por un método complexométrico en que no se procedía a separar previamente la sílice ni los elementos del «grupo de los hidróxidos» (5). Según el primero, debe obtenerse una «alúmina bruta» equivalente a la suma de $\mathrm{Al}_{2} \mathrm{O}_{3}+\mathrm{TiO}_{3}+\mathrm{P}_{2} \mathrm{O}_{5}$, puesto que no se determinaron aparte $\mathrm{TiO}_{2}$ y $\mathrm{P}_{2} \mathrm{O}_{5}$ y, en consecuencia, no se hicieron las correspondientes correcciones; de acuerdo con el segundo, debe obtenerse otra «alúmina bruta», equivalente a la suma $\mathrm{Al}_{2} \mathrm{O}_{3}+\mathrm{TiO}_{2}+\mathrm{Mn}_{3} \mathrm{O}_{3}{ }^{*}$, ya que tampoco se determinaron aparte $\mathrm{TiO}_{3}$ ni $\mathrm{Mn}_{3} \mathrm{O}$, por ello, tampoco se corrigieron los resultados.

Si se tiene en cuenta que, normalmente y por término medio, los contenidos de $\mathrm{P}_{5} \mathrm{O}_{5}$ y de $\mathrm{Mn}_{2} \mathrm{O}_{3}$ en los cementos portland corrientes son similares y del orden de $0,05 \%$ (4), los resultados de «alúmina bruta» obtenidos por ambos métodos deben ser comparables y prácticamente iguales. Estos resultados son los obtenidos, según el primer método, por diferencia entre la suma $\mathrm{Al}_{2} \mathrm{O}_{3}+\mathrm{Fe}_{2} \mathrm{O}_{\mathrm{a}}+\mathrm{TiO}_{2}+\mathrm{P}_{2} \mathrm{O}_{\mathrm{n}}$ hallada por gravimelría y $\mathrm{Fe}_{2} \mathrm{O}_{7}$, determinado por dicromatometría; según el segundo método, por diferencia entre la suma $\mathrm{Al} . \mathrm{O}_{3}+\mathrm{Fe}_{2} \mathrm{O}_{3}+\mathrm{Mn}_{2} \mathrm{O}_{3}{ }^{\circ}$ hallada por complexometria y $\mathrm{Fe}_{2} \mathrm{O}_{3}$ determinado por dicromatometría.

\footnotetext{
* Ya queda Indtcado que la formación del complejo EDTA-Mn no es cuantitativa (6).
} 
Los valores encontrados se exponen en los cuadros 1 y 2 , e indican que los resultados gravimétricos y complexométricos, con diferencias de signo vario y valores absolutos comprendidos dentro de las tolerancias de las Normas A.S.T.M. (1), son perfectamente comparables y equivalentes.

CUADRO NUM. 1

CUADRO NUM. 2

\begin{tabular}{|c|c|c|c|c|c|c|c|}
\hline $\begin{array}{l}\text { Cemento } \\
\text { núm. }\end{array}$ & $\underset{I}{\text { Gravimetria }}$ & $\begin{array}{c}\mathbf{A}_{2} \mathbf{O}_{3}(\%) \\
\text { Complexa- } \\
\text { metria } \\
\text { II }\end{array}$ & $\begin{array}{l}\text { Difereneias } \\
\text { II-1 }\end{array}$ & $\begin{array}{l}\text { Cemento } \\
\text { nứm. }\end{array}$ & Gravimetria & $\begin{array}{l}\mathrm{Al}_{2} \mathrm{O}_{3}(\%) \\
\begin{array}{c}\text { Complexo- } \\
\text { metría } \\
\text { II }\end{array}\end{array}$ & $\begin{array}{l}\text { Diferencias } \\
\text { II-I }\end{array}$ \\
\hline 1 & 6,72 & 6,67 & $-0,05$ & 8 & 5,55 & 5,64 & $+0,09$ \\
\hline 2 & 6,65 & 6,68 & $+0,03$ & 9 & 6,45 & 6,30 & $-0,15$ \\
\hline 3 & 5,49 & 5,58 & $+0,09$ & 10 & 6,60 & 6,61 & $+0,01$ \\
\hline 4 & 7,24 & 7,19 & $-0,05$ & 11 & 6,32 & 6,12 & $-0,20$ \\
\hline 5 & 5,64 & 5,62 & $-0,02$ & 12 & 7,54 & 7,70 & $+0,16$ \\
\hline 6 & 7,53 & 7,25 & $-0,28$ & 13 & 5,14 & 4,99 & $-0,15$ \\
\hline 7 & 5,40 & 5,21 & $-0,19$ & 14 & 6,16 & 6,16 & 0,00 \\
\hline Media & - & - & $-0,07$ & Media & . - & - & $-0,03$ \\
\hline
\end{tabular}

\section{Tercera serie}

Se efectuaron determinaciones de alúmina en cementos, tanto por el método gravimétrico preconizado por las Normas A.S.T.M. (1), como por otro método complexométrico (10), en que se procedió previamente a separar la sílice, a precipitar los elementos del grupo del hidróxido amónico, filtrar, lavar y redisolver el precipitado en ácido clorhídrico y filtrar de nuevo.

De acuerdo con los resultados de la primera serie de experiencias, en estas condiciones no se disuelve el titanio que, por lo tanto, queda separado del aluminio y del hierro. En una parte

CUADRO NUM. 3

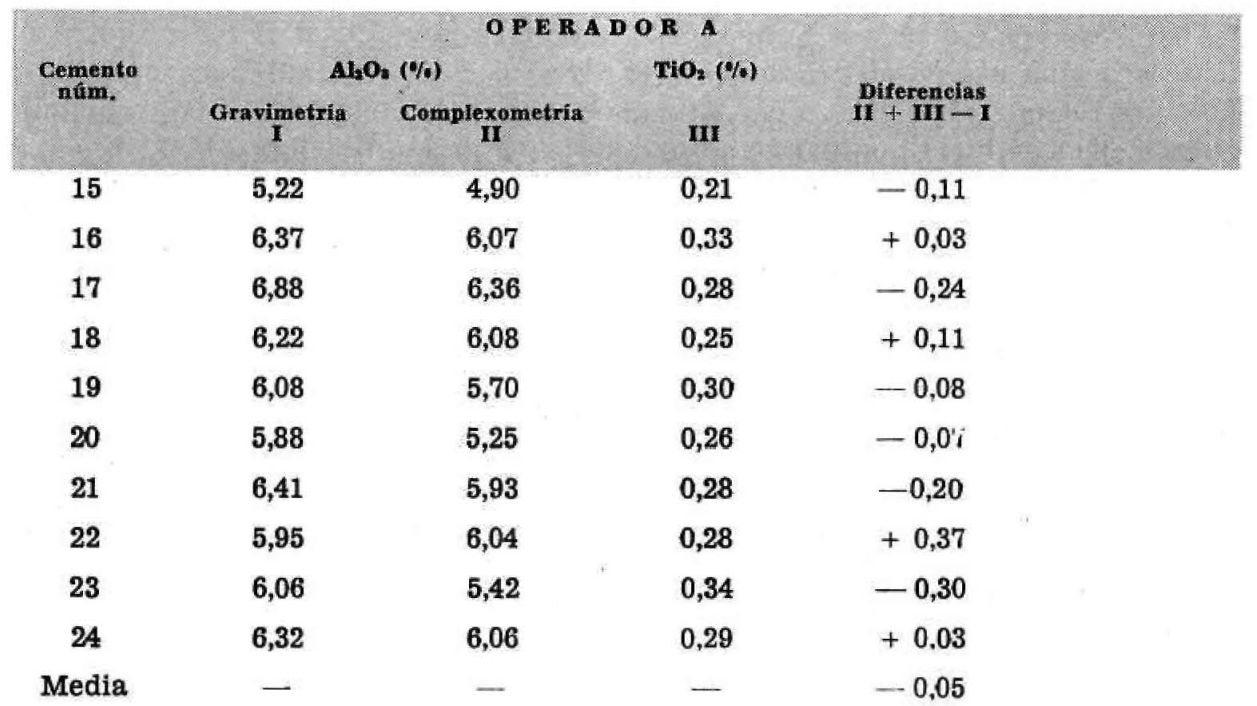


CUADRO NUM. 4

\begin{tabular}{|c|c|c|c|c|c|c|c|c|}
\hline \multirow[b]{2}{*}{$\begin{array}{c}\text { Cemento } \\
\text { núm. }\end{array}$} & \multicolumn{3}{|c|}{ ALOS $(\%)$} & \multicolumn{2}{|c|}{$\operatorname{TiO}_{2}(\%)$} & \multicolumn{3}{|c|}{ Diforencias } \\
\hline & $\begin{array}{l}\text { Operador B } \\
\text { Gravimetria } \\
\quad \text { I }\end{array}$ & $\begin{array}{c}\text { Opersdor A } \\
\text { Complexo- } \\
\text { metria } \\
\text { II }\end{array}$ & $\begin{array}{c}\text { Operador C } \\
\text { Gomplero- } \\
\text { metria } \\
\text { III }\end{array}$ & $\underset{\text { IV }}{\text { Opera- }}$ & $\underset{\mathbf{v}}{\text { Opera- }}$ & $I I+I V-I$ & $\mathbf{I I I}+\mathbf{V}-\mathbf{I}$ & $(\mathbf{I I I}+\mathbf{Y})$ \\
\hline 25 & 5,92 & 5,99 & 5,93 & 0,19 & 0,22 & $+0,26$ & $+0,23$ & $-0,06$ \\
\hline 26 & 6,42 & 6,36 & 6,26 & 0,28 & 0,28 & $+0,22$ & $+0,12$ & $-0,10$ \\
\hline 27 & 5,47 & 5,04 & 5,16 & 0,23 & 0,23 & $-0,20$ & $-0,08$ & $+0,12$ \\
\hline 28 & 9,15 & 8,38 & 8,22 & 0,28 & 0,28 & $-0,49$ & $-0,65$ & $-0,16$ \\
\hline 29 & 6,65 & 6,18 & 6,23 & 0,47 & 0,29 & 0,00 & $-0,13$ & $+0,05$ \\
\hline 30 & 6,19 & 6,05 & 6,29 & 0,34 & - & $+0,20$ & - & - \\
\hline 31 & 5,01 & 4,76 & 4,78 & 0,30 & - & $+0,04$ & - & - \\
\hline 32 & 5,47 & 5,01 & 5,10 & 0,28 & - & $-0,18$ & 一 & - \\
\hline 33 & 5,13 & 5,25 & 5,24 & 0,28 & - & $+0,30$ & $\rightarrow$ & - \\
\hline 34 & 6,19 & 5,86 & 6,05 & 0,25 & - & $-0,08$ & - & - \\
\hline Medias & 一 & - & - & - & - & $+0,01$ & $-0,05$ & $-0,03$ \\
\hline
\end{tabular}

CUADRo NUM. 5

\begin{tabular}{|c|c|c|c|c|c|c|c|c|c|c|}
\hline \multirow{3}{*}{$\begin{array}{c}\text { Cennento } 10 \\
\text { Determinación } \\
\text { núm. }\end{array}$} & \multicolumn{4}{|c|}{$\mathrm{Al}_{2} \mathrm{O}_{2}(\%)$} & \multicolumn{2}{|c|}{$\mathrm{TiO}_{2}(\%)$} & \multicolumn{4}{|c|}{ Diferencias } \\
\hline & \multirow{2}{*}{\multicolumn{2}{|c|}{$\begin{array}{c}\text { Operador A } \\
\text { Gravinie- Complexo- } \\
\text { tria } \\
\text { metria } \\
\text { II }\end{array}$}} & \multicolumn{2}{|c|}{ Opersdor B } & \multirow{2}{*}{$\begin{array}{c}\text { Operador } \\
\mathbf{A} \\
\mathbf{v}\end{array}$} & \multirow{2}{*}{$\begin{array}{c}\text { Operador } \\
\mathbf{B} \\
\mathbf{v}\end{array}$} & \multirow[b]{2}{*}{$11+v-1$} & \multirow[b]{2}{*}{$|W+V|-\mid I I$} & \multirow[b]{2}{*}{$\begin{array}{l}\{(W+Y \mid\} \\
=[H+V\}\end{array}$} & \multirow[b]{2}{*}{$\| \mid 1-1$} \\
\hline & & & $\underset{\substack{\text { Gravime- } \\
\text { tría } \\
\text { III }}}{ }$ & $\begin{array}{c}\text { Compleso- } \\
\text { metría } \\
\text { IV }\end{array}$ & & & & & & \\
\hline 1 & 6,48 & 5,92 & 6,41 & 5,92 & 0,35 & 0,35 & & & & \\
\hline 2 & 6,67 & 5,90 & 6,17 & 5,94 & 0,29 & 0,35 & & & & \\
\hline 3 & 6,22 & 5,73 & 6,30 & 5,70 & 0,35 & 0,35 & & & & \\
\hline 4 & 6,50 & 5,88 & 6,42 & 5,85 & 0,35 & 0,35 & & & & \\
\hline 5 & 6,28 & $\mathbf{5 , 8 8}$ & 7,10 & 5,70 & 0,35 & 0,35 & & & & \\
\hline Medias & 6,43 & 5,86 & 6,48 & 5,82 & 0,34 & 0,35 & $-0,23$ & $-0,31$ & $-0,08$ & $+0,05$ \\
\hline
\end{tabular}

CUADRO NU.Y. 6

\begin{tabular}{|c|c|c|c|c|c|c|c|}
\hline \multirow{2}{*}{$\begin{array}{c}\text { Cemento } \\
\text { N.B.S. 17y } \\
\text { Determinaclon } \\
\text { núm. }\end{array}$} & \multicolumn{4}{|c|}{ 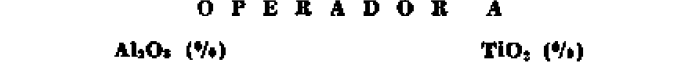 } & \multicolumn{3}{|c|}{ 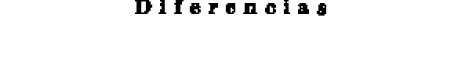 } \\
\hline & $\underset{\mathbf{I}}{\operatorname{Gravimetria}}$ & $\begin{array}{c}\text { Complezo- } \\
\text { metrifia }\end{array}$ & III & IV & $\stackrel{(I I+I V)}{-(I+I I)}$ & $\mathbf{I I}+\mathbf{I I I}-\mathbf{I}$ & II-I \\
\hline 1 & 5,18 & 5,20 & 0,27 & 0,28 & & & \\
\hline 2 & $5, \mathbf{4 1}$ & 5,24 & 0,26 & 0,28 & & & \\
\hline 3 & 5,45 & 5,25 & 0,29 & 0,30 & & & : \\
\hline 4 & 5,37 & 5,00 & 0,30 & 0,28 & & & \\
\hline $\mathbf{5}$ & 5,17 & 5,14 & 0,28 & 0,26 & & & \\
\hline Medias & 5,32 & 5,17 & 0,28 & 0,28 & $-0,15$ & $-0,15$ & $-0,15$ \\
\hline N. B. $\mathbf{S}$. & 5,27 & $(5,17)$ & 0,26 & 0,26 & $(-0,10)$ & $(-0,10)$ & $(-0,10)$ \\
\hline Diferencias. & $+0,05$ & $(0,00)$ & $+0,02$ & $+0,02$ & $(-0,05)$ & $(-0,5)$ & $(-0.05)$ \\
\hline
\end{tabular}

* Alúmine como $\mathrm{Al}_{2} \mathrm{O}_{2}\left(\sin \mathrm{TiO}_{2}\right.$ ). 
del filtrado se determinó por complexometría la suma $\mathrm{Al}_{2} \mathrm{O}_{3}+\mathrm{Fe}_{2} \mathrm{O}_{3}+\mathrm{Mn}_{2} \mathrm{O}_{3}$ ", puesto que el fósforo no forma complejo, y en otra $\mathrm{Fe}_{2} \mathrm{O}_{3}$ por dicromatometría, igual que en el caso anterior.

Los valores encontrados se exponen en los cuadros 3 a 6 que incluyen resultados gravimétricos y complexométricos de varios operadores, relativos a distintos cementos, $O$ a varias determinaciones hechas con un mismo cemento.

Prácticamente, en la totalidad de los casos, las diferencias entre los resultados complexométricos «corregidos», es decir, expresados por la suma $\mathrm{Al}_{2} \mathrm{O}_{3}$ complexométrico más $\mathrm{TiO}_{2}$ (con la eventual aunque poco probable inclusión parcial de $\mathrm{Mn}_{2} \mathrm{O}_{3}{ }^{\text {so }}$ ) y los resultados gravimétricos expresados por una «alúmina bruta», suma de $\mathrm{Al}_{2} \mathrm{O}_{3}, \mathrm{TiO}_{2}$ y $\mathrm{P}_{2} \mathrm{O}_{5}$, entran dentro de las tolerancias admitidas por las Normas A.S.T.M. Esto sucede, tanto para resultados de un mismo operador como de operadores distintos, y tanto al comparar valores complexométricos «corregidos» con gravimétricos, como complexométricos o gravimétricos entre sí. Las diferencias encontradas, no sólo están dentro de las tolerancias de las citadas Normas, sino que en todos los casos son del mismo orden de magnitud. Se observa, en general, una tendencia de los valores graviméiricos «corregidos» de la alúmina, es decir, disminuidos en el tanto por ciento de $\mathrm{TiO}_{3}$, a ser muy poco superiores a los complexométricos, muy probablemente porque los primeros incluyen $\mathrm{P}_{2} \mathrm{O}_{5}$ que no incluyen los segundos.

Por lo tanto, el método complexométrico tiene, aparte de sus ventajas de rapidez y sencillez, como volumétrico que es, las de ser tan preciso y reproducible como el gravimétrico clásico, y dar un valor para la alúmina $\left(\mathrm{Al}_{2} \mathrm{O}_{3}\right)$ mucho más ajustado a la realidad y más apto para utilizar en el cálculo de la composición potencial del cemento, sin necesidad de tener que determinar aparte $\mathrm{TiO}_{2}$ ni $\mathrm{P}_{2} \mathrm{O}_{4}$ por vía de corrección.

\section{Blbllografía}

(1) A.S.T.M. Standards C 114-61, Parte 4 (1961).

(2) Bosue, R. H.: "The Chemistry of Portland Cement". Reinhold Publ. Co., 2." ed. New York (1955).

(3) TreadWeld, F. P.: "Tratado de Química Analítica". Tomo I. Análisis Cualitativo. M. Marín, Barcelona (1958)

(4) N.B.S. (National BURead of Standards): Certificate of Analyses: Standara Sample 177 (Portland Cement). Wáshington (1957).

(5) Callejh, J., y Framántez Paris, J. M.: "Determinación complexcmétrica de la alúmina en el cemento", Revista de Ciencia Aplicada, núm. 69, 326-333 (1959).

(6) BARONE, P., y Frigrone, R.: "Determinazione complessometrica dzll'alluminio in presenza di manganeso", L'Industria Ital. del Cemento, 31/9, 439-446 (1961).

(7) FlaschKa, $H_{4}$ " "EDTA Titrations". Pergamon Press, London-New York (1969).

(8) Bermejo, F., y Prieto, A.: "Aplicaciones analíticas del AEDT y análogos", pág. 260. Imprenta Seminario Conciliar, Santiago de Compostela (1960).

(9) Bersaejo, F., y PRIETo, A.: "Aplicaciones analíticas del AEDT y análogos", pág. 411. Imprenta Seminario Conciliar, Santiago de Compost zla (1980).

(10) Calleja, J., y Fgrnádezz Paris, J. M.: "Manual de Analisis Complexométrico del Cemento Portland". I. E. T. c. c., en vias de publicacion (1963).

\footnotetext{
* Ya queda 1ndicado que la formación del complejo EDTA-Mn no es cuantitativa (6).

* Las pequefias cantidades de manganeso que puedan formar complejo con el EDTA en las condiciones de trabajo, en la generalidad de los casos no desvirtúan los resultados (6).
} 